\title{
Algebraic Analysis of a Rabin-Like Cryptosystem and Its Countermeasures
}

\author{
Muhammad Asyraf Asbullah' and Muhammad Rezal Kamel Ariffin ${ }^{2}$ \\ 'Al-Kindi Cryptography Research Laboratory, Institute for Mathematical Research (INSPEM), \\ Universiti Putra Malaysia; \\ ${ }^{2}$ Mathematics Department, Faculty of Science, Universiti Putra Malaysia;
}

\begin{abstract}
Objective: In this paper, we present two algebraic analyses upon a new Rabin-like public key cryptosystem namely the Rabin- $p$ cryptosystem. Methods/Analysis: We show that by using the continued fraction's method and the Coppersmith's theorems, there exists inappropriate parameter's size that can affect the security of Rabin- $p$ cryptosystem. Findings: The first analysis proved that the prime factors of its public key can be found amongst the list of the continued fraction expansion of the ciphertext $c$ and the modulus $N=p^{2} q$ in polynomial time. For the second analysis, by using the Coppersmith's theorems we showed that the message $m$ can be retrieved in polynomial time provided some condition on the message length. We also propose a countermeasure to avoid both analyses. Novelty/Improvement: The purpose of this work is to offer suggestions for a countermeasure for the aforementioned analysis upon implementing the Rabin- $p$ cryptosystem. Hence, all the parameters should be chosen carefully.
\end{abstract}

Keywords: Algebraic Analysis, Continued Fraction, Coppersmith’s Theorems, Rabin- $p$ Cryptosystem

\section{Introduction}

Post-1990's, the exponentially growing common medium for transporting information is through electronic means. Currently, the internet assumes this role. Today, our daily activities and conversation are dependent on the internet connectivity. With just simple clicks, we could send emails or communicate with people, do monetary transactions through electronic commerce, and purchase items online. Out of the wilderness and all the sophistication that we experience within the online world, the field of cryptography turns into a handy tool when security begins to matters. Cryptography provides a mean to ensure that our privacy and confidential information is secured, hence provides confidence for sharing and exchanging such information between other parties (the sender and the intended receiver). It is of a great interest, to be able to analyze the strengths and weaknesses of encryption and decryption processes.
The very first public key cryptosystem was introduced in 1978 which is widely recognized as the RSA cryptosystem ${ }^{11}$. One year after the invention of the Rivest, Shamir, and Adelman (RSA) cryptosystem, Rabin cryptosystem ${ }^{9}$ introduced another cryptosystem based on the intractability to solve the square root modulo problem of a composite integer. In fact, this cryptosystem is the first public key cryptosystem of its kind that comes with a security that was proven as hard as factoring.

Basically, in comparison, the Rabin encryption is faster than the RSA cryptosystem and it is proven to be equivalent to factoring while the RSA is not ${ }^{10}$. Theoretically speaking, the Rabin cryptosystem is better than the RSA, but the question is why the Rabin cryptosystem is not being commercialized as the RSA? The main reasons are because the Rabin decryption produces four-to-one output, which leads to decryption failure, and the equivalence relation to factoring lead to a security breach for the Rabin cryptosystem ${ }^{4}$. Efforts were made by numerous

${ }^{*}$ Author for correspondence 
scholars to solve Rabin four-to-one decryption problem. However, their efforts may reduce the encryption speed or do not hold the equivalence relation to factoring, or may have a probability of decryption failure.

The Rabin cryptosystem was introduced more than 30 years ago. Since then, it was revised and revisited by many scholars, in order to make it as implementable as the RSA. As a consequence, we witnessed that there are a lot of Rabin-like cryptosystems in existence. A good recent survey on the variants of Rabin cryptosystem can be found in Asbullah and Ariffin ${ }^{\circ}$. Interestingly, this survey shows that the study based on the Rabin cryptosystem is still an active research topic. Recently, a new Rabin-like cryptosystem namely the Rabin- $p$ cryptosystem was proposed ${ }^{8}$. Such cryptosystem is purposely designed without using the Jacobi symbol, redundancy in the message and avoiding the demands of extra information for finding the correct plaintext. Decryption outputs a unique plaintext without any decryption failure. In addition, Rabin- $p$ decryption only requires a single prime number. Moreover, and computes a single modular exponentiation instead of two modular exponentiation executed by other Rabin variants. As a result, this reduces the computational effort during the decryption process.

In this paper, we apply two types of analysis upon a new Rabin-like public key cryptosystem namely the Rabin- $p$ cryptosystem. We show that by using the continued fraction's method and the Coppersmith's theorems, there exists inappropriate parameter's size that can affect the security of Rabin- $p$ cryptosystem. The first analysis proved that the prime factors of its public key can be found amongst the list of the continued fraction expansion of the ciphertext $c$ and the modulus $N$ in polynomial time. For the second analysis, by using the Coppersmith's theorems we showed that the message $m$ can be retrieved in polynomial time provided some condition on the message length. The aim of this work is to offer suggestions for a countermeasure for the aforementioned cryptanalysis upon implementing the Rabin- $p$ cryptosystem. Hence, all the parameters should be chosen carefully.

The remainder of the paper is organized as follows. In Section 2, we present a few basic facts about the continued fraction algorithm and the Coppersmith's method for solving modular polynomial equations. In Section 3, we present the algebraic analysis using the continued fraction's method and the Coppersmith's theorems upon the
Rabin- $p$ cryptosystem. We also propose a countermeasure to avoid both analyses. Finally, we conclude in Section 4 .

\section{Preliminaries}

This section is structured as follows. We start with the description of the Rabin- $p$ cryptosystem, followed by a brief recap of basic facts about the continued fraction algorithm and Coppersmith's method.

\subsection{Rabin- $p$ Cryptosystem ${ }^{8}$.}

In this section, we provide the description of Rabin- $p$ cryptosystem proposed by Asbullah and Ariffin ${ }^{8}$ as follows. We first describe the Rabin- $p$ key generation, encryption and decryption procedures. The key generation algorithm of the Rabin- $p$ cryptosystem (Algorithm 1) produces two random and distinct primes $p$ and $p$ of the same length such that $p, q \equiv 3(\bmod 4)$. The key generation algorithm then produces an integer $N=p^{2} q$, which is denoted as the public key. The private key is the prime $\mathrm{p}$.

\section{Algorithm 1: Rabin-p Key Generation Algorithm}

INPUT: The size $k$ of the prime numbers.

OUTPUT: The public key $N=p^{2} q$ and the private key $p$

1. Generate random and distinct $K$-bit primes $p q$ such that $p, q \equiv 3(\bmod 4)$ where $2^{\mathrm{k}}<p, q<2^{\mathrm{k}+1}$.

2. Compute $N=p^{2} q$

3. Return the public key $N$ and a private key $P$

\author{
Algorithm 2: Rabin- $p$ Encryption Algorithm \\ INPUT: The plaintext $c$ and the public key $N$ \\ OUTPUT: A ciphertext $m$. \\ 1. Choose plaintext $0<m<2^{2 \mathrm{k}+1}$ such that $\operatorname{gcd}(\mathrm{m}, \mathrm{N})=1$ \\ 2. Compute $\mathrm{c}=\mathrm{m}^{2}(\bmod N)$. \\ 3. Return the ciphertext $c$.
}

\section{Algorithm 3: Rabin- $p$ Decryption Algorithm}

INPUT: A ciphertext $c$ and the private key $p$

OUTPUT: The plaintext $m$
1. Compute $\mathrm{c}=\mathrm{m}^{2}(\bmod N)$
2. Compute $m_{p} \equiv c^{\frac{p+1}{4}}(\bmod p)$ 
3. Compute $i \equiv \frac{c-m_{p}^{2}}{p}$

4. Compute $j \equiv \frac{i}{2 m_{p}}(\bmod p)$

5. Compute $m_{1}=m_{p}+j p$

6. If $m_{1}<2^{\mathbf{2 k - 1}}$, then return $m=m_{1}$. Else, return $m=p^{2}-m_{1}$

\subsection{Continued Fraction}

In this section, we give the basic definition of continued fractions as follows. Observe that the theory of continued fractions is one of the main methods used in the study of algebraic analysis upon a public key cryptosystem. For instance, see $e^{2,3,7,7}$.

Theorem 1 (Legendre's Theorem) ${ }^{6}$.

Let $R$ is a rational number. Let $\in \mathbb{Z}, y \neq 0$ and $\operatorname{gcd}(x, y)=1$. Suppose $\left|R-\frac{x}{y}\right|<\frac{1}{2 y^{2}}$, then $\frac{x}{y}$ is a convergent of the continued fraction expansion of $R$.

\subsection{Coppersmith's Theorem}

In general, finding solutions to modular equations is easy if we know the factorization of the modulus. Else, it can be difficult. Consequently, a significantly powerful method for finding small roots of modular polynomial equations was invented which called as the Coppersmith's theorem $^{5}$. When working with modulo of a prime number, there is no reason to use the Coppersmith's theorem since there exist far better root-finding algorithm (for instance, Newton method), yet in cryptography, we usually deal with a number of the product of primes ${ }^{12}$. Moreover, this method has found many different applications in the area of cryptography and a vastly useful tool for cryptanalysis 2 . We immediately provide the Coppersmith's theorem as follows.

\section{Theorem 2 (Coppersmith)}

Let $N$ be an integer of unknown factorization. Let $f_{N}(x)$ be a univariate, a monic polynomial of degree $\delta$. Then we can find all solutions $x_{0}$ for the equation $f_{N}(x) \equiv 0(\bmod N)$

with $\left|x_{0}\right|<N^{\frac{1}{\delta}}$ in polynomial time.

\section{Theorem 3 (May) 1}

Let $N$ be an integer of unknown factorization, which has a divisor $b>N^{\beta}$. Furthermore, let $f_{b}(x)$ be a univariate, a monic polynomial of degree $\delta$. Then we can find all solutions $x_{0}$ for the equation $f_{N}(x) \equiv 0(\bmod N)$ with

$\left|x_{0}\right|<\frac{1}{2} N \frac{\beta^{2}}{\delta}$ in polynomial time.

\section{Results and Analysis}

In this section, we show the algebraic analysis using the continued fraction's method and the Coppersmith's theorems, respectively.

\subsection{Continued Fraction's Method}

Suppose $C$ and $N$ are the parameters from the Rabin- $p$ cryptosystem. Since we have the ciphertext $c(\bmod N)$, thus $c<N$. Therefore ${ }^{c}$ can be written as $c=a+b p q$ or $c=a^{\prime}+b^{\prime} p^{2}$ for some integer $a, a^{\prime}, b, b^{\prime}$.

\section{Theorem 4}

Let $c=a+b p q$ for some positive integer $a$ and $b$.

If $a<\frac{q}{2}$ and $b<p$, then $\frac{b}{p}$ can be found amongst the list of the continued fraction expansion of $\frac{C}{N}$. Furthermore, $N$ can be factored in polynomial time.

Proof: Consider the value $c=a+b p q$ and $N=p^{2} q$. if we divide $c=a+b p q$ by $N$ then we obtain $\frac{c}{N}=\frac{a+b p q}{N}=\frac{a}{N}+\frac{b p q}{N}=\frac{a}{N}+\frac{b}{p}$. Rearranging, we have $\frac{c}{N}-\frac{b}{p}=\frac{a}{N}$. Thus, to show that $\left|\frac{c}{N}-\frac{b}{p}\right|<\frac{1}{2 p^{2}}$, it suffice if $\frac{a}{N}<\frac{1}{2 p^{2}}$, which implies that $a<\frac{N}{2 p^{2}}=\frac{q}{2}$. Hence, by Theorem $1, \frac{b}{p}$ is a convergent of the continued 
fraction expansion of $\frac{c}{N}$ if $a<\frac{q}{2}$. This leads to finding the prime factors $p$ and then $q$

\section{Theorem 5}

Let $c=a^{\prime}+b^{\prime} p^{2}$ for some positive integer $a^{\prime}$ and $b^{\prime}$. If $a^{\prime}<\frac{p^{2}}{2 q}$ and $b^{\prime}<q$, then $\frac{b^{\prime}}{q}$ can be found amongst the list of the continued fraction expansion of $\frac{C}{N}$. Furthermore, $N$ can be factored in polynomial time.

Proof: Consider the value $c=a^{\prime}+b^{\prime} p^{2}$ and $N=p^{2} q$. if we divide $c=a^{\prime}+b^{\prime} p^{2}$ by $N$ then we obtain

$\frac{c}{N}=\frac{c=a^{\prime}+b^{\prime} p^{2}}{N}=\frac{a^{\prime}}{N}+\frac{b^{\prime} p^{2}}{N}=\frac{a^{\prime}}{N}+\frac{b^{\prime}}{q}$.

Rearranging, we have $\frac{c}{N}-\frac{b^{\prime}}{q}=\frac{a^{\prime}}{N}$. Thus, to show that

$\left|\frac{c}{N}-\frac{b^{\prime}}{q}\right|<\frac{1}{2 q^{2}}$, it suffice if $\frac{a^{\prime}}{N}<\frac{1}{2 q^{2}}$, which implies

that $a^{\prime}<\frac{N}{2 q^{2}}=\frac{p^{2}}{2 q}$. Hence, by Theorem $1, \frac{b^{\prime}}{q}$ is a con-

vergent of the continued fraction expansion of $\frac{C}{N}$ if

$a^{\prime}<\frac{p^{2}}{2 q}$. This leads to finding the prime factors $q$ and then $p$.

\section{Remark 1}

Consider the analysis result from Theorem 4. As a suggestion for the countermeasure, in order to avoid such attack, the variable $a \equiv c(\bmod p q)$ should be checked carefully

such that $a>\frac{q}{2}$. While, from Theorem 5, the variable $a^{\prime} \equiv c\left(\bmod p^{2}\right)$ should be checked carefully such that $a^{\prime}>\frac{p^{2}}{2 q}$.

\subsection{Coppersmith's Method}

We now analyze the Rabin- $p$ cryptosystem based on the Theorem 2 and Theorem 3 and obtain the following results. Suppose $c, m$ and $N$ are the parameters from the Rabin- $p$ cryptosystem.

Theorem 6
Let $c \equiv m^{2}(\bmod N)$ and $N=p^{2} q$.If $m<2^{\frac{3 k}{2}}$, then $m$ can be found in polynomial time.

Proof: Suppose $c \equiv m^{2}(\bmod N)$ and $N=p^{2} q$

. Consider the univariate, monic polynomial $f_{N}(x) \equiv x^{2}-c \equiv 0(\bmod N)$. By applying Theorem 2, we set $\delta=2$. Hence the root $x_{0}=m$ can be recovered if $m<N^{\frac{1}{\delta}}=N^{\frac{1}{2}} \approx 2^{\frac{3 k}{2}}$.

\section{Theorem 7}

Let $c \equiv m^{2}\left(\bmod p^{2}\right)$ such that $p^{2}$ is an unknown factor for $N$.If $m<2^{\frac{2 k}{3}}$, then $m$ can be found in polynomial time.

Proof: Suppose $c \equiv m^{2}\left(\bmod p^{2}\right)$ such that $p^{2}$ is an unknown factor for $N$. Consider the univariate, monic polynomial $f_{p^{2}}(x) \equiv x^{2}-c \equiv 0\left(\bmod p^{2}\right){ }_{\text {with }}$ $p^{2} \approx N^{\frac{2}{3}} \approx 2^{2 k}$. By applying Theorem 3 , we can find a solution $\operatorname{root} x_{0}=m$ if $m<\frac{1}{2} N^{\frac{\beta^{2}}{\delta}}<N^{\frac{2}{9}} \approx 2^{\frac{2 k}{3}}$.

\section{Remark 2}

Consider the analysis result from Theorem 6 and Theorem 7. As a suggestion for countermeasure, in order to avoid both attacks, we would set the message

$m>2^{\frac{3 k}{2}}$ in the Rabin- $p$ encryption algorithm.

\section{Conclusion}

We now summarize the result of the paper. Firstly, we showed the algebraic analysis upon the Rabin- $p$ cryptosystem using the continued fraction's method and the Coppersmith's theorems. The continued fraction method apply when $<\frac{q}{2}$

and $b<p$ (or $a^{\prime}<\frac{p^{2}}{2 q}$ and $b^{\prime}<q$ ) satisfies an 
equation $c=a+b p q$ (or $\left.c=a^{\prime}+b^{\prime} p^{2}\right)$, then $\frac{b}{p}$

$b$

(or $\bar{q}$ ) is a convergent of the continued fraction expansion

$C$

of $\bar{N}$. We also analyze the Rabin- $p$ cipher text using the

Coppersmith's theorems and produce two possible ways

to recover the plaintext $m$ whenever satisfies $m<2^{\frac{3 k}{2}}$

or $m<2^{\frac{2 k}{3}}$. Therefore, as a countermeasure we would

set $m>2^{\frac{a k}{2}}$ in the Rabin- $p$ encryption algorithm.

\section{References}

1. May A. New RSA vulnerabilities using lattice reduction methods [Doctoral dissertation]. Germany, University of Paderborn; 2003 Oct 19. p. 6-151.

2. Nitaj A. Diophantine and lattice cryptanalysis of the RSA cryptosystem. In Artificial Intelligence, Evolutionary Computing and Metaheuristics, Springer Berlin Heidelberg; 2013. p. 139-68.

3. Nitaj A. A new vulnerable class of exponents in RSA. JP Journal of Algebra, Number Theory and Applications. 2011; 21:203-20.
4. Boneh D. Simplified OAEP for the RSA and Rabin functions. In Annual International Cryptology Conference Springer Berlin Heidelberg; 2001 Aug 19. p. 275-91.

5. Coppersmith D. Small solutions to polynomial equations, and low exponent RSA vulnerabilities. Journal of Cryptology. 1997 Sep 1; 10(4):233-60.

6. Hardy GH, Wright EM. An introduction to the theory of numbers. Oxford University Press; 1979.

7. Asbullah MA, Ariffin MR. New attacks on RSA with modulus $\mathrm{N}=\mathrm{p} 2 \mathrm{q}$ using continued fractions. Journal of Physics: Conference Series, IOP Publishing. 2015; 622(1):012019.

8. Asbullah MA, Ariffin MR. Design of Rabin-like cryptosystem without decryption failure. Malaysian Journal of Mathematical Sciences. 2016 August; 10(S):1-18.

9. Rabin MO. Digitalized signatures and public key cryptosystems as intractable as factorization. MIT/LCS/TR-212, Technical Report MIT; 1979.

10. Koblitz N, MenezesAJ. Another look at provable security. Journal of Cryptology. 2007 Jan 1; 20(1):3-7.

11. Rivest RL, Shamir A, Adleman L. A method for obtaining digital signatures and public-key cryptosystems. Communications of the Association for Computing Machinery (ACM). 1978 Feb 1; 21(2):120-6.

12. Galbraith SD. Mathematics of public key cryptography. Cambridge University Press; 2012 Mar 15. 